\title{
Transport and magnetotransport properties across the two-step Verwey transition in $\mathrm{BaGdFe}_{2} \mathrm{O}_{5+w}$
}

\author{
J. Lindén \\ Department of Physics, Åbo Akademi, FIN-20500 Turku, Finland \\ P. Karen \\ Department of Chemistry, University of Oslo, Blindern N-0315 Oslo, Norway
}

J. Nakamura, M. Karppinen, and H. Yamauchi

Materials and Structures Laboratory, Tokyo Institute of Technology, Yokohama 226-8503, Japan

(Received 3 October 2005; revised manuscript received 15 December 2005; published 10 February 2006)

\begin{abstract}
Magnetoresistance, electrical transport, and magnetic properties associated with a two-step Verwey-type transition in $\mathrm{BaGdFe}_{2} \mathrm{O}_{5+w}(-0.015<w<0.181)$ are studied as a function of temperature and oxygen nonstoichiometry $(w)$. A disproportionation of $\mathrm{Fe}^{2.5+}$ into $\mathrm{Fe}^{2.5-\epsilon}$ and $\mathrm{Fe}^{2.5+\epsilon}$ upon cooling through the transition temperature $T_{\mathrm{p}}$ and charge ordering into $\mathrm{Fe}^{2+}$ and $\mathrm{Fe}^{3+}$ at $T_{\mathrm{V}}$ are manifested in electrical-conductivity and Seebeck-coefficient data. Above $T_{\mathrm{V}}$, electrical conductivity shows an activated hopping behavior with activation energy of $\sim 0.13 \mathrm{eV}$. Seebeck measurements identify holes as charge carriers below $T_{\mathrm{V}}$. Above $T_{\mathrm{V}}$, both holes and valence-mixing electrons need to be considered, although the Seebeck coefficient remains positive up to room temperature. This suggests that the activation energy for electrons is higher than that for holes, and the actual value is close to that obtained from conductivity data. Increasing $w$ increases electrical conductivity and decreases Seebeck coefficient in the charge-ordered state. In the valence-mixed state, increasing $w$ increases Seebeck coefficient, but conductivity increases only up to $w \approx 0.1$, from which the decay of the valence mixing takes over and conductivity begins to approach values extrapolated from the charge-ordered state. Magnetoresistance peaks with negative ratio up to $\sim 2 \%$ are observed, corresponding to a small magnetic-susceptibility change at $T_{\mathrm{V}}$.
\end{abstract}

DOI: 10.1103/PhysRevB.73.064415

PACS number(s): 72.20.Pa, 72.20.My, 75.30.Mb

\section{INTRODUCTION}

Whereas in colossal-magnetoresistance manganese oxides charge ordering occurs on the background of either ferromagnetic (FM) or antiferromagnetic (AFM) interactions, ${ }^{1-4}$ double-cell perovskites $\mathrm{BaRFe}_{2} \mathrm{O}_{5}(R=$ a rare-earth element) order iron charges within an overall AFM arrangement. ${ }^{5} \mathrm{Un}-$ paralleled by any similar transition, this charge ordering phenomenon proceeds in two steps, in two separate transitions. The first step upon cooling below a temperature $T_{\mathrm{p}}$ is characterized by separation of $\mathrm{Fe}^{2.5+}$ into intermediate charges $\mathrm{Fe}^{2.5-\epsilon}$ and $\mathrm{Fe}^{2.5+\epsilon}$. This separation takes place in the structural frame of the slight orthorhombic distortion associated with an AFM order, in which the iron spins are aligned along the shortest bond. ${ }^{6}$ The second step which accomplishes the full charge separation into equal amounts of the $\mathrm{Fe}^{2+}$ and $\mathrm{Fe}^{3+}$ species, ${ }^{5}$ takes place at a lower temperature $T_{\mathrm{V}}$. This second transition proceeds in parallel with the Verwey transition in magnetite, ${ }^{7}$ exhibiting abrupt changes in entropy, electrical conductivity and volume. ${ }^{6}$ Although a long-range ordering of the fully separated valence and spin states of iron has been structurally characterized by neutron powder diffraction, ${ }^{6,8}$ no such order could be detected ${ }^{8}$ in the temperature range of the intermediate valence states $\mathrm{Fe}^{2.5-\epsilon}$ and $\mathrm{Fe}^{2.5+\epsilon}$ between the two phase transitions.

Such double-cell perovskites are subject to a considerable oxygen nonstoichiometry of the $\mathrm{Ba} \mathrm{Fe}_{2} \mathrm{O}_{5+w}$ type, where the vacant oxygen site, located in the $R$ layer, is partially filled by the added oxygen atoms $w$. The nonstoichiometry has a dramatic effect on the concentration of valence-mixed species, removing them faster than the nominal concentration of divalent Fe would predict. ${ }^{5}$ Upon increasing $w$, the intermediate states, $\mathrm{Fe}^{2.5-\epsilon}$ and $\mathrm{Fe}^{2.5+\epsilon}$, become soon no longer observable as the weaker first step of the charge separation is eliminated, and the structure adopts a long-range tetragonal symmetry imposed by disordered arrangement of the added oxygens. This overrides the magnetostrictive orthorhombic distortion of the ideal structure with $w=0 .{ }^{8}$ The remaining single-step transition is rather robust as a first-order transition and disappears only at nonstoichiometry levels as high as $w=0.25 .^{9,10}$

Ferromagnetic magnetite has been reported to exhibit generic magnetoresistance which changes from positive above $T_{\mathrm{V}}$ to negative below. ${ }^{11,12}$ A negative magnetoresistance effect was observed for a nearly stoichiometric composition of $\mathrm{BaSmFe}_{2} \mathrm{O}_{5.0} \cdot{ }^{13}$ In this work, the effect of the oxygen nonstoichiometry on electrical conductivity, magnetic susceptibility, thermoelectric power, and magnetoresistance (MR) are studied on single-phase and well-characterized samples.

\section{EXPERIMENT}

Syntheses. $\mathrm{BaGdFe}_{2} \mathrm{O}_{5+w}$ was synthesized by a wetchemical technique via citrate-based nanoprecursor. The details of the procedure are described in Ref. 9, here just in brief: Standardized starting materials, gadolinium oxide 
(>99.9\%, Molycorp), iron lumps (99.95\%, Koch-Light, dissolved in a diluted high-purity nitric acid), and $\mathrm{BaCO}_{3}(0.1 \%$ $\mathrm{Sr}$, Merck, dried at $300{ }^{\circ} \mathrm{C}$ ) were dissolved in the said order in melted high-purity citric acid monohydrate. The clear orange-brown melt was dehydrated into a porous solid at $180{ }^{\circ} \mathrm{C}$, milled and incinerated at $450{ }^{\circ} \mathrm{C}$. The obtained amorphous powder was calcined at $900{ }^{\circ} \mathrm{C}$ for $20 \mathrm{~h}$ in the atmosphere of $\mathrm{Ar}$ and $\mathrm{H}_{2}$ in a volume ratio of 100:1 saturated by water vapor to $p_{\mathrm{H}_{2} \mathrm{O}}=0.0215 \mathrm{bar}$. The single-phase brown-colored calcinate product was milled in a vibration agate mill, cold pressed at 160 bars and sintered into a fulldensity master-sample at $1010{ }^{\circ} \mathrm{C}$ in the atmosphere of $\mathrm{Ar}$ and $\mathrm{H}_{2}$ premixed in the volume ratio of 17.7(1):1 and saturated to have $p_{\mathrm{H}_{2} \mathrm{O}}=0.02488$ bar. The oxygen content was controlled by a quenching technique that is described in detail in Ref. 6, together with the details of the cerimetric determination of the oxygen content. The latter has reproducibility better than \pm 0.003 . It should be kept in mind, however, that this value represents an overall average oxygen content and that a certain distribution of oxygen contents occurs in the equilibrated and quenched samples, with half width of some $w=0.010 .^{9}$

Magnetic and magnetotransport properties. Temperature dependences of magnetic susceptibilities of all samples were measured upon warming and cooling in constant fields of 0.01 and $50 \mathrm{kOe}$. For the least nonstoichiometric sample, magnetization was measured as a function of the applied field and temperature across the range of the Verwey-type transition.

For magnetotransport measurements the compact samples were cut under a protective layer of ethyleneglycole into parallelepipeds with a cross section of $2-3 \mathrm{~mm}^{2}$. Measurements were performed under an applied field of -7 to $+7 \mathrm{~T}$ (fieldscanning mode), in a direction perpendicular to the current flow, within temperature range of 150-300 K (temperaturescanning mode), with a Quantum Design PPMS-9 instrument.

Electrical transport properties. A standard four-probe technique was used, with gold electrode pads vacuumdeposited directly on the sample surface. The spacing between the electrodes was controlled by a $0.05-\mathrm{mm}$-thin $\mathrm{Au}$ mask, to which gold leads were attached using an $\mathrm{Ag}$ paste.

Seebeck measurements. Seebeck coefficient was evaluated from measurements of the thermoelectric effect. Copper plates were attached to the two opposite largest faces of parallelepipeds of dimensions $0.3 \times 1.5 \times 2.0 \mathrm{~mm}^{3}$ with the help of $\mathrm{Ag}$ paste. One of the plates was heated resistively so that a temperature difference of $\Delta T \leqslant 2.0 \mathrm{~K}$ was maintained between the ends of the sample, measured by thermocouples. The overall temperature was varied from 290 to $210 \mathrm{~K}$, while the thermovoltage between the plates was measured using a nanovoltmeter device (HP 34420A). For each temperature, several thermovoltage readings were collected by adjusting the power of the resistive heater. The Seebeck coefficient was obtained from the slope of the voltage versus $\Delta T$.

\section{RESULTS AND DISCUSSION}

\section{A. Characterization}

The master $\mathrm{BaGdFe}_{2} \mathrm{O}_{5+w}$ sample was single-phase according to synchrotron powder x-ray diffraction. The non-

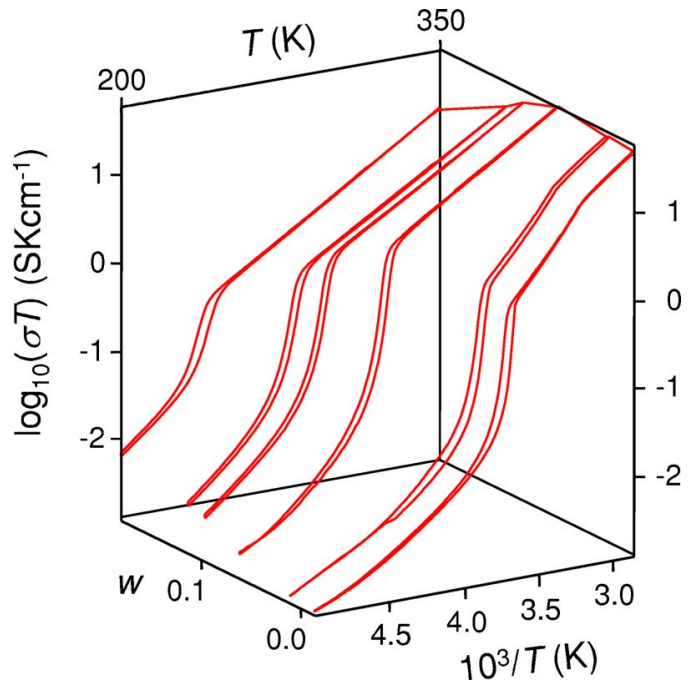

FIG. 1. (Color online) Temperature and composition dependence of electrical conductivities of $\mathrm{BaGdFe}_{2} \mathrm{O}_{5+w}$ samples $(w$ $=-0.015,0.010,0.061,0.096,0.113,0.181)$ around Verwey-type transition under ambient magnetic field. Axes correspond to linearity for a small-polaron hopping model, see text.

stoichiometry $w$ was controlled in a range adjacent to the ideal mixed-valence composition having $w=0$, and this included also a sample with a slight excess of divalent iron, $w=-0.015$, according to a cerimetric titration. Upon further reduction, metallic iron is detected in such samples by $\mathrm{x}$-ray powder diffraction and is manifested also by ferromagnetic properties. Having shown very weak magnetic properties of such a type, but without the direct proof by conventional x-ray powder diffraction, this sample is assumed to contain traces of metallic Fe. Since the next more oxidized sample with $w=-0.001$ did not show the ferromagnetic behavior, it is assumed that the $w=-0.001$ sample is within the lower homogeneity limit with respect to the oxygen nonstoichiometry $(w \approx 0.000)$.

\section{B. Electrical conductivity under ambient field}

The characteristic abrupt change in electrical conductivity at $T_{\mathrm{V}}$ in $\mathrm{BaGdFe}_{2} \mathrm{O}_{5+w}$ is shown in Fig. 1 as a function of oxygen nonstoichiometry. The magnitude of this change decreases in parallel with the demise of this discontinuous transition, as quantified for example by diminishing $\Delta S$ or $\Delta V$ at $T_{\mathrm{V}} \cdot{ }^{9} \quad$ Upon increasing $w$, electrical conductivity of $\mathrm{BaGdFe}_{2} \mathrm{O}_{5+w}$ approaches a value that is lower than that of the stoichiometric $(w=0)$ valence-mixed phase (above $\left.T_{\mathrm{V}}\right)$, but higher than that of the stoichiometric charge-ordered phase (below $T_{\mathrm{V}}$ ). The isothermal variations as a function of nonstoichiometry in the two regions are profoundly different: From the charge-ordered state with $w=0$, electrical conductivity increases monotonously with increasing $w$ [the left face of the three-dimensional (3D) plot in Fig. 1], as expected for increased doping of the localized $\mathrm{Fe}^{2+}$ and $\mathrm{Fe}^{3+}$ stoichiometric states present when $w=0$. From the valencemixed state having $w=0$ (the right face of the 3D plot in Fig. 1), electrical conductivity passes through a maximum 
TABLE I. Transition temperatures $T_{\mathrm{V}}$ and $T_{\mathrm{p}}$ upon cooling, as extracted from conductivity data for $\mathrm{BaGdFe}_{2} \mathrm{O}_{5+w}$ in Fig. 1.

\begin{tabular}{rrr}
\hline \hline$w$ & $T_{\mathrm{p}}(\mathrm{K})$ & $T_{\mathrm{V}}(\mathrm{K})$ \\
\hline-0.015 & 313.2 & 270.2 \\
0.010 & 311.9 & 270.0 \\
0.019 & 304.2 & 265.5 \\
0.061 & & 249.6 \\
0.096 & & 239.5 \\
0.113 & & 234.6 \\
0.181 & & 226.2 \\
\hline
\end{tabular}

around $w=0.100$ before it eventually descends towards the level of the heavily doped phase. At the maximum, the concentration of the valence-mixed atoms (according to Mössbauer data $\left.{ }^{5}\right)$ is approximately one half the level at $w=0$.

For the two compositions that are closest to the ideal stoichiometric situation $(w=-0.015$ and 0.010 ; the front face of Fig. 1), a two-step change in electrical conductivity is clearly seen in Fig. 1, related to the two-step course of the Verweytype transition: A kink corresponding to the partial charge ordering is followed by the more conspicuous abrupt change of the Verwey transition proper at lower temperature. Only the latter transition is clearly discontinuous, showing also a hysteresis. Transition temperatures are listed in Table I as idealized onsets of electrical conductivity change upon cooling, determined as intersections of the high-temperature trend with the tangent drawn at the inflexion point of the transient curve.

As illustrated by Mössbauer spectroscopy, ${ }^{5}$ there is a variety of valence and spin states of iron that may produce carriers contributing to electrical conductivity of $\mathrm{BaGdFe}_{2} \mathrm{O}_{5+w}$. In addition to the charge-ordered, or partially charge-ordered, or valence-mixed iron states, there remains a small amount of the nonmixed regular integer-valence states of high-spin $\mathrm{Fe}^{2+}$ and high-spin $\mathrm{Fe}^{3+}$, even at the stoichiometric composition with $w=0$. The oxygen loading $(w)$ supplies high-spin $\mathrm{Fe}^{3+}$ at the expense of all the other states. Nevertheless, some general features can be extracted. The temperature-dependence curves of electrical conductivity show an activated behavior that changes character when $T_{\mathrm{V}}$ is crossed, but remains in principle the same as a function of $w$.

Below $T_{\mathrm{V}}$, the electrical transport approaches a variablerange hopping mechanism, given that a reasonable linearity against $T^{-1 / 4}$ is observed towards the lowest temperature limits. Such behavior is often observed for localized electron states.

In the fully valence-mixed range, an Arrhenius-type activation gives a reasonable linear fit, which further slightly improves with the application of a diffusion-assisted hopping model:

$$
\sigma=\left(\frac{e N_{c}}{a_{\mathrm{sp}}^{3}}\right) \mu \exp \left[-\frac{E_{\mu}}{k_{\mathrm{B}} T}\right],
$$

where the mobility $\mu$ is given by:

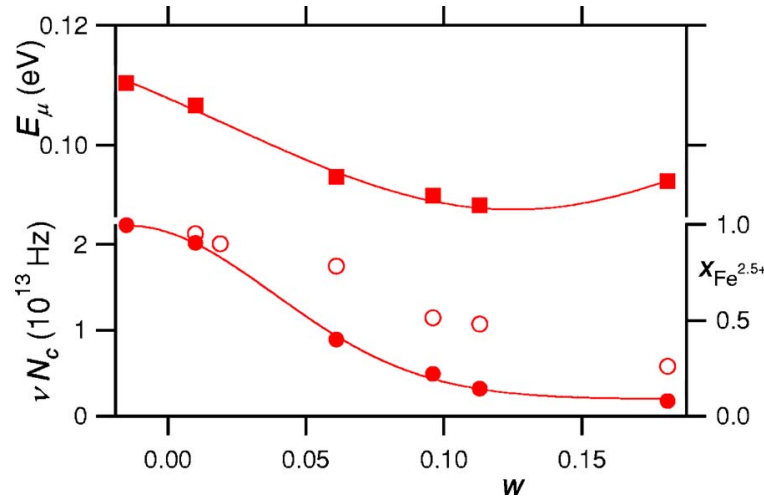

FIG. 2. (Color online) Parameters of the small-polaron hopping model of electrical conductivity in valence-mixed $\mathrm{BaGdFe}_{2} \mathrm{O}_{5+w}$. Top: Activation energy for $\left(E_{\mu}\right)$ conductivity (full symbols) Bottom: The product of hopping frequency and amount of charge carriers $\left(\nu N_{c}\right.$, full symbols). For comparison, the relative decrease in the concentration of the Mössbauer $\mathrm{Fe}^{2.5+}$ component $\left(x_{\mathrm{Fe}^{2.5+}}\right)$ (Ref. 14) is plotted in open symbols.

$$
\mu=e h_{p} a_{s p}^{2} \frac{\nu}{k_{\mathrm{B}} T}
$$

In Eqs. (1) and (2) $h_{p}$ is the hopping probability, $N_{c}$ the number of carriers per single-perovskite cell, $a_{s p}$ the average edge of such a cell, $e$ the electron charge, $k_{\mathrm{B}}$ the Boltzmann constant, $E_{\mu}$ the activation energy, and $\nu$ the hopping frequency. Assuming that the hopping occurs between neighboring iron sites, $a_{s p}$ is approximated as the edge of the average single-perovskite cubic unit cell of the phase, calculated from the room-temperature unit-cell parameters. Assuming further that the hopping probability is $h_{p}=1 / 5$ (instead of the usual 1/6 for the octahedral site), $E_{\mu}$ and $\nu N_{c}$ are extracted as two parameters of the least-squares fitting to the experimental data.

Energy $E_{\mu}$ has a minimum for compositions about $w$ $=0.12$ that corresponds to the maximum in conductivity shown in Fig. 1 (the right face of the cube). Around this level of oxygen nonstoichiometry, the concentration of the valence-mixed state is still high enough to make a significant contribution to conductivity, while the ratio between the formally di- and tri-valent iron ions becomes increasingly favorable for percolations of the valence-mixed $\mathrm{Fe}^{2.5+}$ pairs via random fluctuations between alternative neighbors $\mathrm{Fe}^{2+}$ and $\mathrm{Fe}^{3+} \cdot 5$ In fact, this maximum in conductivity (and minimum in activation energy) corresponds to the maximum in product of the concentration of the valence-mixed state and the amount of available choices of di- and tri-valent iron pairs as a function of $w$ [Eq. (5) and Fig. 16 in Ref. 5].

The electrical conductivity data do not provide infomation on the sign and the concentration of charge carriers. An estimate of changes in the concentration of carriers $N_{c}$ as a function of $w$ can be done for the valence-mixed region, but only under assumption that the hopping frequency $\nu$ is constant. As shown in Fig. 2, the $\nu N_{c}$ curve roughly correlates to the deterioration of valence mixing as a function of $w$. However, this merely reflects the fact that the increase in electri- 


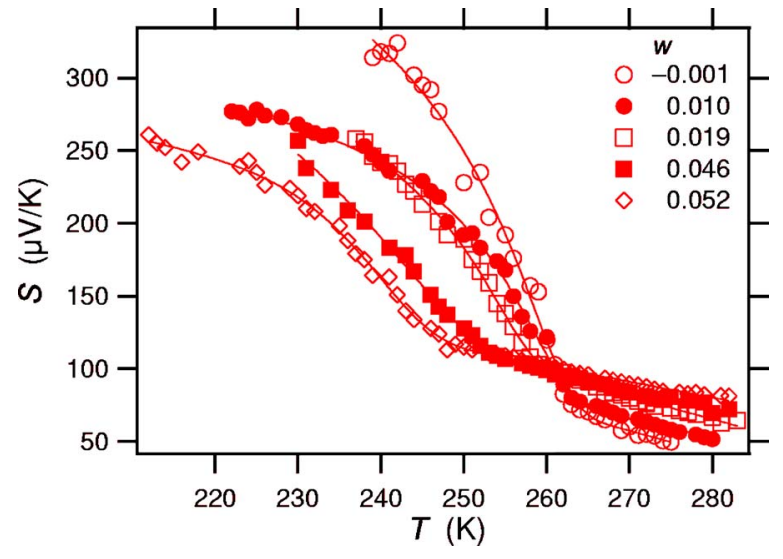

FIG. 3. (Color online) Temperature dependence of Seebeck coefficient for $\mathrm{BaGdFe}_{2} \mathrm{O}_{5+w}$.

cal conductivity above $T_{\mathrm{V}}$ is directly or indirectly caused by the valence mixing.

\section{Seebeck effect measurements}

In order to obtain the sign and the properties of carriers, measurements of thermoelectric effect have been performed with respect to temperature and $w$, the latter in the vicinity of $w=0$ where the manifestation of the two-step Verwey transition is the strongest. Seebeck coefficient data $(S)$ are displayed in Fig. 3. Although the data at lower temperatures and higher values of $S$ were increasingly difficult to measure, the general features in Fig. 3 lead to several important conclusions: (i) The positive sign of $S$ throughout seems to indicate that the charge carriers are holes, or that at least holes give a large contribution. (ii) The concentration of charge carriers increases ( $S$ decreases) with increasing temperature, showing that we are in general beyond the $T^{1 / 2}$ dependence typical of variable-range hopping and rather in the region of activated hopping. (iii) Heating the sample through the transition affects the number of carriers in a similar rather abrupt manner as the electrical resistance (note that according to a powder $\mathrm{x}$-ray diffraction study $^{9}$ the Verwey transition proper proceeds over an interval of some $15-20 \mathrm{~K}$ ). (iv) The effect of oxygen nonstoichiometry $(w)$ on $S$ is inverted across the $T_{\mathrm{V}}$.

\section{Valence-mixed state}

The dominance of holes as carriers seems to contradict the understanding of the valence mixing as electron sharing between the Fe sites. One would imagine that the very same electrons act as charge carriers. However, one could infer that the electron which is shared between two Fe atoms is not delocalized over the lattice, because a transfer to a nearby lattice site in the $a b$ plane would require a spin-flip due to the antiferromagnetic coupling of the lattice. Through activation such a transfer may be possible, though.

As the oxygen content is increased, the number of holes should increase. This leads to a problem: if the Seebeck coefficient depends only on the hole concentration, then the increase in $S$ upon increasing $w$ is just opposite to what is expected. We will therefore assume that both holes and elec- trons contribute to the Seebeck coefficient, i.e., that we are dealing with a bipolar case. As the Seebeck coefficient was measured in zero-current mode and the carrier mobilities can hence be neglected, we may employ the following expression: ${ }^{15}$

$$
S=\frac{n S_{n}+p S_{p}}{n+p},
$$

where $n$ and $p$ represent concentrations of electrons and holes, and $S_{n}$ and $S_{p}$ are the Seebeck coefficients for electrons and holes. Both carriers would be thermally activated at relatively low teperatures, with activation energies $E_{n}$ and $E_{p}$ :

$$
S_{n}=-\frac{E_{n}}{e T}
$$

and

$$
S_{p}=\frac{E_{p}}{e T} .
$$

The Seebeck coefficient and conductivity data suggest that the source of the conducting electrons is the valence mixing. The number of the valence-mixing electrons per $\mathrm{Fe}$ is equal to one half of the mole fraction of $\mathrm{Fe}^{2.5+}$, which can be registered by Mössbauer spectroscopy and follows a probability-based equation derived in Ref. 5:

$$
x_{\mathrm{Fe}^{2.5+}}=2(0.5-w)\left[1-(0.5-w)^{4}\right] \frac{0.5-w}{0.5+w} .
$$

The number of thermally activated electrons per Fe atom is then

$$
n=\frac{x_{\mathrm{Fe}^{2.5+}}}{2} \exp \left(-E_{n} / k_{\mathrm{B}} T\right) .
$$

Since $\mathrm{Fe}^{2+}$ would not be easily accommodated at the rather compressed octahedral sites formed by the added oxygen $w$, only pentacoordinated $\mathrm{Fe}^{3+}$ is considered to act as holes, hence

$$
p=\left(0.5-\frac{x_{\mathrm{Fe}^{2.5+}}}{2}\right) \exp \left(-E_{p} / k_{\mathrm{B}} T\right),
$$

where $0.5-x_{\mathrm{Fe}^{2.5+}+} / 2$ is the mole fraction of pentacoordinated $\mathrm{Fe}^{3+}$; obtained by subtracting the fraction of pentacoordinated iron that went into valence mixing from the constant concentration 0.5 that would be present if the valence mixing did not take place.

The Seebeck coefficent data above $T_{\mathrm{V}}$ are fitted with Eq. (3) and $E_{p}, E_{n}$ as parameters (Fig. 4). These activation energies are plotted in Fig. 5 as a function of $w$ and compared with the activation energy for conductivity, showing a reasonably good agreement.

The following conclusions can now be drawn: Even when the number of electrons exceeds the number of holes for low $w$ values, the Seebeck coefficient is dominated by the holes, due to the fact that the activation energy for the electrons is approximately five times larger. This in turn seems to follow from the fact that the electrons are practically localized be- 


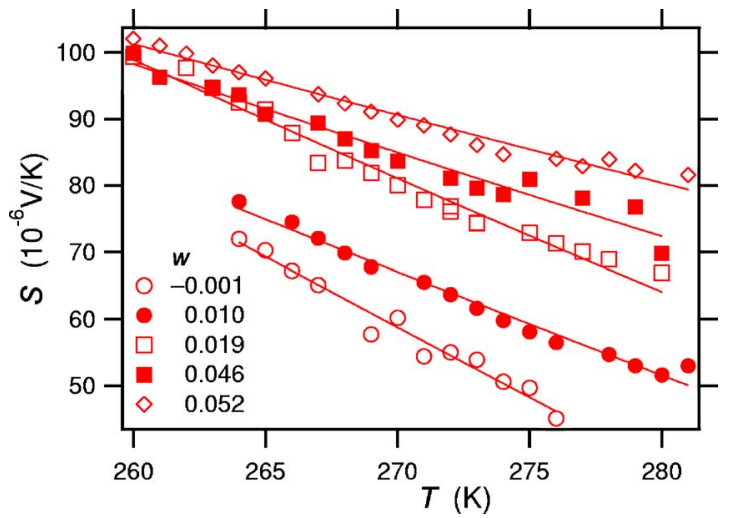

FIG. 4. (Color online) Seebeck coefficients for $\mathrm{BaGdFe}_{2} \mathrm{O}_{5+w}$ fitted using Eq. (3) in the region $T>T_{\mathrm{V}}$.

tween two Fe atoms along the $c$ axis and can only be moved with a costly spin-flip process. The holes, which act as $p$ type impurities are readily activated. Upon increasing temperature, negative Seebeck coefficients would eventually be expected, as more and more electrons become activated. This is also apparent from extrapolaton of data in Fig. 3.

The data in Fig. 2 suggest that the concentration of charge carriers as a function of $w$ follows closely the number of mixing electrons given by the Mössbauer data [see also Eq. (6)]. This means that the contribution of holes to electric conductivity is small, which in turn must be due to the fact that the mobility of the electrons, once they are activated, is much larger than that of the holes. This would make the electrons to dominate in the measured conductivity. The thermoelectric power being an essentially zero-current measurement does not depend on the carrier mobilities and thus the holes should dominate the Seebeck coefficient value. The huge decrease in conductivity when temperature is lowered below $T_{\mathrm{V}}$ can now be explained: when the electrons are completely localized, holes remain as charge carriers, and the overall conductivity decreases because of their low mobility. The fact that the hole contribution to conductivity is twoorders of magnitudes smaller than the electron contribution also justifies the use of a single-carrier model for the conductivity data.

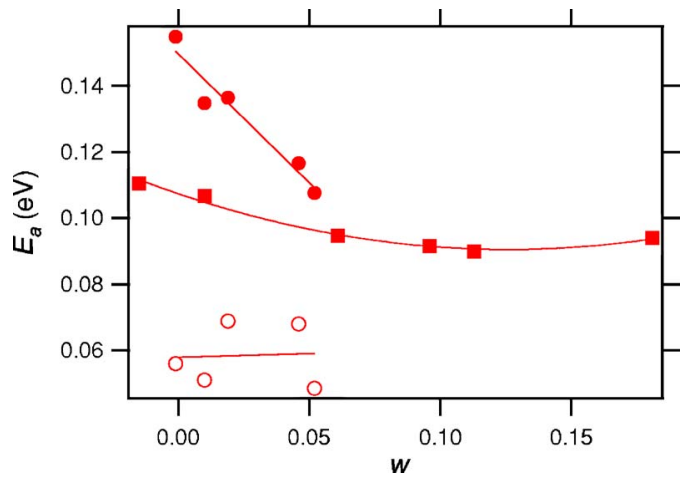

FIG. 5. (Color online) Activation energies of electrons (filled circles) and holes (open circles) in $\mathrm{BaGdFe}_{2} \mathrm{O}_{5+w}$, as obtained from fitting the $T>T_{\mathrm{V}}$ Seebeck data with Eq. (3). For comparison activation energies obtained from conductivity data (Fig. 2) are plotted (filled squares).

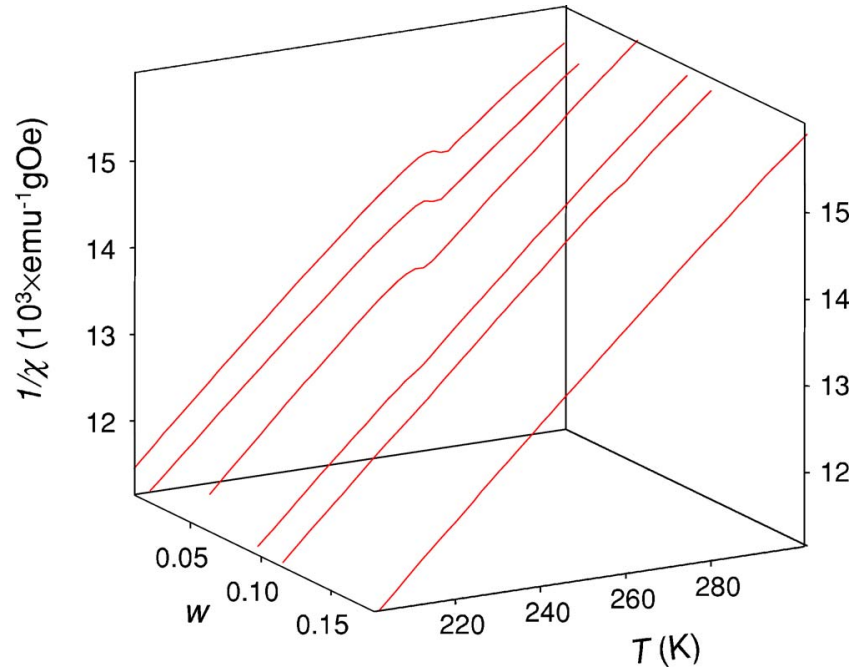

FIG. 6. (Color online) Temperature and composition dependence of the inverse magnetic susceptibility of $\mathrm{BaGdFe}_{2} \mathrm{O}_{5+w}(w$ $=-0.015,0.010,0.061,0.096,0.113,0.181)$, in the temperature range covering $T_{\mathrm{V}}$, under a magnetic field of $50 \mathrm{kOe}$.

\section{Charge-ordered state}

Below $T_{\mathrm{V}}$, the structure is charge ordered, and the valence-mixing electrons become completely localized. The Seebeck coefficient decreases with increasing $w$, suggesting a pure hole conductor in which holes originate from the oxygen nonstoichiometry and are carried by the same species as above $T_{\mathrm{V}}$ : pentacoordinate $\mathrm{Fe}^{3+}$ defects in the ideal chargeordered structure. These defects are formed via oxidation by the added oxygens $w$.

The Seebeck data were difficult to measure at low temperatures below $T_{\mathrm{V}}$ and temperature dependences could not be obtained for all samples. This prevented us from systematic fitting of the entire Seebeck data with Eq. (5) common for holes above and below $T_{\mathrm{V}}$ and Eq. (4) for electrons above $T_{\mathrm{V}}$.

Data for samples with $w=0.010$ and 0.052 (Fig. 3) suggest that there would be a good agreement for such a joint model for the Seebeck coefficients below and above $T_{\mathrm{V}}$ : the fitted values for $E_{p}$ when inserted in Eq. (5) yield positive Seebeck values of the correct magnitude $(\sim 200 \mu \mathrm{V} / \mathrm{K})$ and trend with respect to $w$.

\section{Magnetic susceptibility}

All samples exhibit strong paramagnetism due to the presence of $\mathrm{Gd}^{3+}$ moments. Upon this background, magnetization changes of iron atoms at the Verwey transition appear weak. However, under strong fields it is possible to observe an increase in susceptibility right above $T_{\mathrm{V}}$. As illustrated in Fig. 6, the magnitude of this jump decreases rapidly with increasing oxygen nonstoichiometry. Since powder neutrondiffraction data ${ }^{6,8,16}$ show only a negligible change in the magnitude of the ordered AFM moment across $T_{\mathrm{V}}$ in $\mathrm{BaRFe}_{2} \mathrm{O}_{5+w}$, the observed susceptibility jump (at $T_{\mathrm{V}}$ ) suggests that the exchange interactions are weaker in the valence mixed phase. The size of the magnetization jump as a func- 


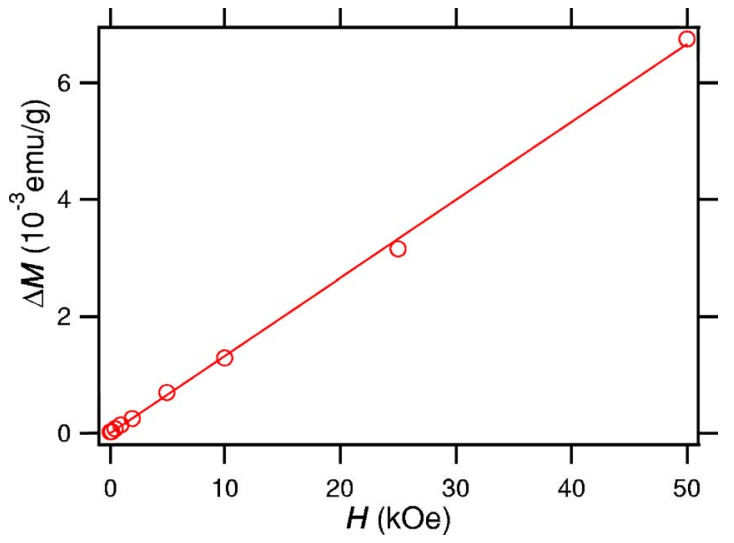

FIG. 7. (Color online) Change in specific magnetization of $\mathrm{BaGdFe}_{2} \mathrm{O}_{5.010}$ upon warming through $T_{\mathrm{V}}$ as a function of the external field.

tion of the field, evaluated in Fig. 7, illustrates a linear dependence without any tendency to approach saturation. A careful analysis of the field-dependent data shows that $T_{\mathrm{V}}$ decreases by $\sim 1 \mathrm{~K}$ per $50 \mathrm{kOe}$.

\section{E. Magnetoresistivity}

The resistivity versus temperature behavior under applied fields up to $7 \mathrm{~T}$ revealed MR effects. However, due to hysteresis, resistivity at a given temperature changes considerably when it is remeasured under various external fields. As a result, MR peaks observed in the temperature scans may even exhibit positive values, i.e., such scans merely serve to indicate the position of the peak. In order to get reliable magnetoresistance data, field scans at constant temperatures were performed. In Fig. 8, the general trend in MR ratios obtained from such field scans is illustrated.

Figure 9 shows that both the premonitory transition (when present) and the main transition give rise to (negative) MR peaks. The MR peaks reach a maximum around $w=0$ and decay as oxygen nonstoichiometry is increased (Fig. 10).

Since both the magnetization jump and the MR ratio at $T_{\mathrm{V}}$ are nearly linear functions of the external field $H$ (Figs. 7 and

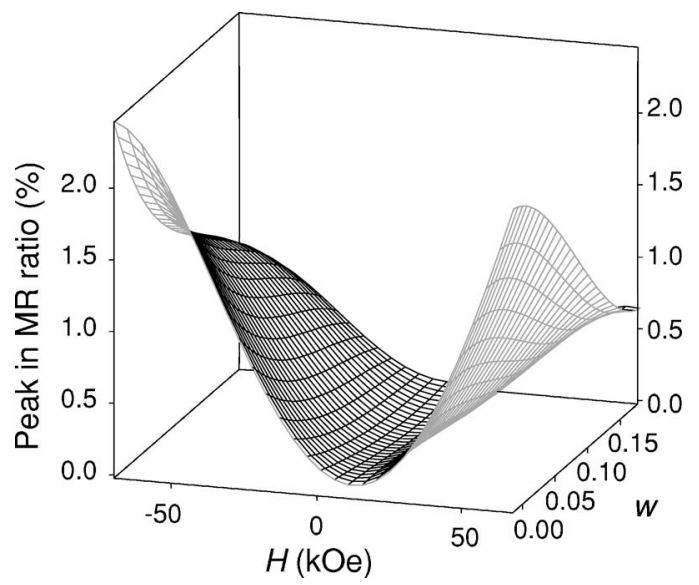

FIG. 8. Fitted surface of MR ratios at $T_{\mathrm{V}}$ versus external magnetic field and nonstoichiometry parameter $w$ in $\mathrm{BaGdFe}_{2} \mathrm{O}_{5+w}$.

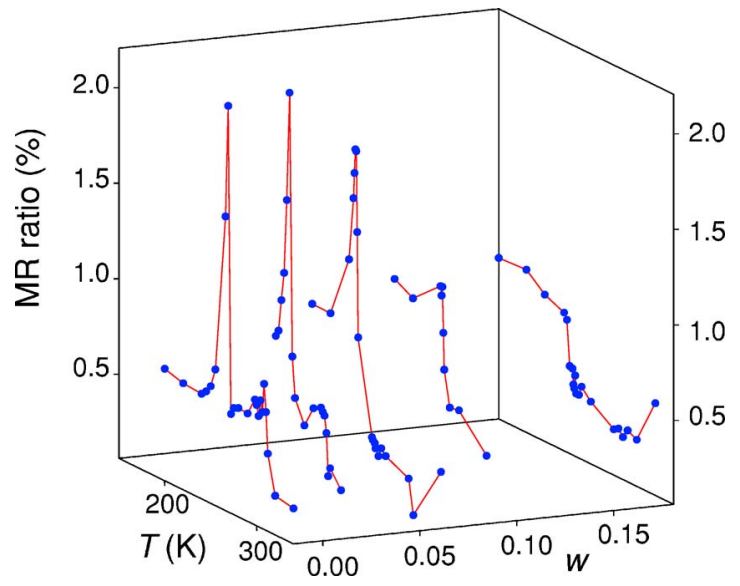

FIG. 9. (Color online) The negative MR ratios versus temperature for $\mathrm{BaGdFe}_{2} \mathrm{O}_{5+w}$, obtained from field scans at constant temperatures.

8 respectively), we have also plotted the maximum MR ratio at $T_{\mathrm{V}}$, as obtained from Fig. 10, versus the magnetization jump $(\Delta M / M)$ for several samples. The result is presented in Fig. 11. The MR peak ratios have been scaled by a factor of $5 / 7$, as the maximum field values for the MR and for the magnetization measurements were 7 and $5 \mathrm{~T}$, respectively. This confirms that MR associated with the Verwey-type transition in $\mathrm{BaGdFe}_{2} \mathrm{O}_{5+w}$ is a consequence of the susceptibility change at $T_{\mathrm{V}}$. Any such change at $T_{\mathrm{p}}$ was probably too small to be registered.

\section{CONCLUSIONS}

Studies on the electric transport properties in $\mathrm{BaGdFe}_{2} \mathrm{O}_{5+w}$ revealed an increase in resistivity (up to two orders of magnitude) upon cooling the samples below the charge-ordering transition at $T_{\mathrm{V}}$. Analysis of the Seebeck data showed that above $T_{\mathrm{V}}$ the charge carries consist of both positive holes and electrons. Due to their lower activation energy, holes dominate in the Seebeck coefficent, despite their concentration being lower than that of electrons. At the same time, the valence-mixing electrons control conductivity above $T_{\mathrm{V}}$, owing to their larger mobility. The concentration

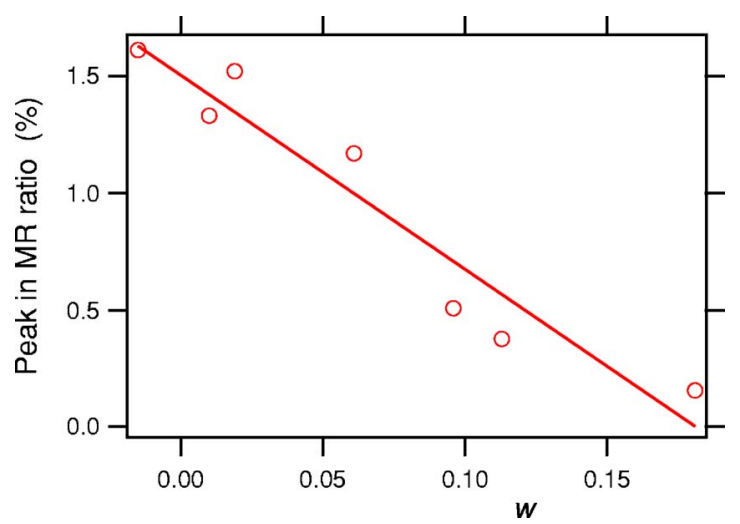

FIG. 10. (Color online) MR peak heights at $T_{\mathrm{V}}$ (background levels of $\sim 0.5 \%$ subtracted) for $\mathrm{BaGdFe}_{2} \mathrm{O}_{5+w}$. 


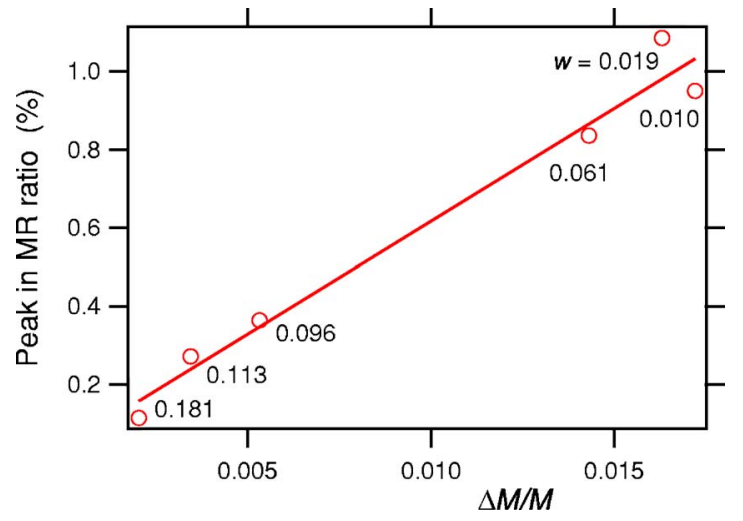

FIG. 11. (Color online) MR peak heights for $\mathrm{BaGdFe}_{2} \mathrm{O}_{5+w}$ at $T_{\mathrm{V}}$ versus change in specific magnetization at $T_{\mathrm{V}}$ in external field of $5 \mathrm{~T}$ (background levels of $\sim 0.5 \%$ subtracted; the MR peak heights rescaled by $5 / 7$, see text).

of holes and electrons determined from the conductivity and the Seebeck coefficient data roughly corresponds to the concentration of excess oxygen $w$ (manifested as holes) and the number of mixed-valence pairs $\mathrm{Fe}^{2.5+}$ (manifested as elec- trons), as obtained from the chemical analyses and Mössbauer spectroscopy data, respectively. The overall low conductivity of the phase and the dominance of holes as activated charge carriers indicate that the $\mathrm{Fe}^{2+}$ and $\mathrm{Fe}^{3+}$ valence mixing above $T_{\mathrm{V}}$ is not a completely delocalized phenomenon. Below $T_{\mathrm{V}}$, the valence-mixing electrons localize completely and electric conductivity is mainly carried by the holes. Accordingly, Seebeck coefficient decreases with increasing $w$. The MR effect of about $2 \%$ is registered at the main charge-ordering transition $\left(T_{\mathrm{V}}\right)$ in stoichiometric $\mathrm{BaGdFe}_{2} \mathrm{O}_{5}$ and a proportionally smaller effect occurs at $T_{\mathrm{p}}$ of the incipient (premonitory) charge ordering. Both decrease in parallel with the removal of the valence-mixed iron states, as oxygen nonstoichiometry is increased.

\section{ACKNOWLEDGMENTS}

J.L. acknowledges grants received from the Scandinavia Sasakawa Foundation and the Magnus Ehrnrooth Fund. This work was supported by Grants-in-aid for Scientific Research (Grant Nos. 15206002 and 15206071) from the Japan Society for the Promotion of Science.
${ }^{1}$ Z. Jirák, S. Krupička, Z. Šimša, M. Dlouhá, and S. Vratislav, J. Magn. Magn. Mater. 53, 153 (1985).

${ }^{2}$ Y. Tomioka, A. Asamitsu, Y. Moritomo, H. Kuwahara, and Y. Tokura, Phys. Rev. Lett. 74, 5108 (1995).

${ }^{3}$ Y. Moritomo, H. Kuwahara, Y. Tomioka, and Y. Tokura, Phys. Rev. B 55, 7549 (1997).

${ }^{4}$ P. M. Woodward, T. Vogt, D. E. Cox, A. Arulraj, C. N. R. Rao, P. Karen, and A. K. Cheetham, Chem. Mater. 10, 3652 (1998).

${ }^{5}$ J. Lindén, P. Karen, A. Kjekshus, J. Miettinen, T. Pietari, and M. Karppinen, Phys. Rev. B 60, 15251 (1999).

${ }^{6}$ P. Karen, P. Woodward, J. Lindén, T. Vogt, A. Studer, and P. Fischer, Phys. Rev. B 64, 214405 (2001).

${ }^{7}$ E. J. W. Verwey, Nature (London) 144, 327 (1939).

${ }^{8}$ P. M. Woodward and P. Karen, Inorg. Chem. 42, 1121 (2003).
${ }^{9}$ P. Karen, J. Solid State Chem. 170, 9 (2003).

${ }^{10}$ P. Karen, P. M. Woodward, P. N. Santhosh, T. Vogt, P. W. Stephens, and S. Pagola, J. Solid State Chem. 167, 480 (2002).

${ }^{11}$ D. Kostopoulos, Phys. Status Solidi A 9, 523 (1972).

${ }^{12}$ M. Ziese and H. J. Blythe, J. Phys.: Condens. Matter 12, 13 (2000).

${ }^{13}$ J. Nakamura, J. Lindén, M. Karppinen, and H. Yamauchi, Appl. Phys. Lett. 77, 1683 (2000).

${ }^{14}$ J. Lindén (unpublished).

${ }^{15}$ Yu. G. Gurevich, O. Yu. Titov, G. N. Logvinov, and O. I. Lyubimov, Phys. Rev. B 51, 6999 (1995).

${ }^{16}$ P. M. Woodward, E. Suard, and P. Karen, J. Am. Chem. Soc. 125, 889 (2003). 